\title{
Stones in A Congenital Ureteric Diverticulum
}

\author{
(1) Oshan Basnayake1, (1) Heshan Siriwardena1, (D) Munipriya Willaraarachchi1, (D) Madura Ambegoda1', (D) Sathiskumar Thevarajah², \\ (D) Anuruddha Abeygunasekera1 \\ 'Department of Urology, Colombo South Teaching Hospital, Dehiwala, Sri Lanka \\ 2Department of Radiology, Colombo South Teaching Hospital, Dehiwala, Sri Lanka
}

\begin{abstract}
True ureteral diverticula are rare, may be found incidentally, or form after urinary sepsis, stones, or obstruction. Stone formation is an uncommon complication because of urine stasis or recurrent infections. Radiological opacities suggestive of a stone in an extra-anatomical site of the ureter should raise the suspicion of a ureteric diverticulum. Although a congenital ureteric diverticulum is believed to be formed because of an abortive ureteric bud, its anatomy could be completely different from that of a duplex ureter. The presence of complications requires diverticulectomy with or without reconstruction of the ureteric defect.
\end{abstract}

Keywords: Ureteral diverticulum, calculi, ureteric duplication, urinary stones

\section{Introduction}

A true ureteral diverticulum is a rare condition. It may be an incidental finding on imaging or associated with symptoms such as hematuria, dysuria, and pain (1). Complications of ureteric diverticula include infection, stones, and obstruction $(2,3)$. A case of a transitional cell carcinoma developing in a ureteral diverticulum has also been reported (4). Herein, we report a patient with a long pelvic ureteral diverticulum containing several calculi.

\section{Case Reports}

A 39-year-old otherwise healthy man attended the urology clinic for evaluation of persistent dysuria. He had been having dysuria for six months, without any other lower urinary tract symptoms, fever, or ureteric colic. His urine analysis showed pyuria, and urine cultures grew coliform bacteria on three occasions. He had been treated with antibiotics according to the sensitivity pattern. His urinary tract ultrasonography was normal, but X-ray kidney, ureter, and bladder (KUB) showed a hyperdense opacity in the pelvis medial to the normal pathway of the left ureter measuring $1 \mathrm{~cm} \times 1.5 \mathrm{~cm}$ (Figure 1a). Computed tomography (CT) urogram showed a possible diverticulum arising from the left side of the distal ureter extending posteromedio-inferiorly and containing two calculi (Figures 1b-d). The patient underwent open surgery, and the left lower ureter was accessed extraperitoneally. The diverticulum was $10 \mathrm{~cm}$ long, and the origin was $2.5 \mathrm{~cm}$ proximal to the vesicoureteric junction. It was extending posteriorly in between the pelvic muscles toward the sacrum and lateral aspect of the mid-rectum. The diverticulum was mobilized fully and removed after ligation at the root (Figure 2a). His postoperative recovery was uneventful. The histopathological examination of the excised diverticulum showed flattened urothelium with atrophy of the muscularis propria of the wall (Figure 2b). Few foci were showing signs of inflammation. The infrared spectroscopic analysis revealed that the stone was composed of calcium oxalate monohydrate and calcium oxalate dihydrate crystals. After three months of follow-up, he was symptom-free, and his urinary tract ultrasonography showed normal upper urinary tracts. Informed written consent for publication of the text and accompanying images was obtained from the patient.

\section{Discussion}

Knowledge on ureteral diverticula is based on case reports and case series because of its rare occurrence. Ureteral diverticula are divided into two types (2). The congenital form usually represents the abortive duplication of the ureter, while the

Correspondence: Oshan Basnayake MD, Department of Urology, Colombo South Teaching Hospital, Dehiwala, Sri Lanka

Phone: +94718446557Ｅ-mail: oshanbasnayake@gmail.com ORCID-ID: orcid.org/0000-0002-1239-7506

Received: $31.01 .2021 \quad$ Accepted: 23.03.2021

Cite this article as: Basnayake 0 , Siriwardena $\mathrm{H}$, Willaraarachchi M, Ambegoda M, Thevarajah S, Abeygunasekera A. Stones in A Congenital Ureteric Diverticulum. J Urol Surg 2021;8(4):300-302.

${ }^{\circ}$ Copyright 2021 by the Association of Urological Surgery / Journal of Urological Surgery published by Galenos Publishing House. 

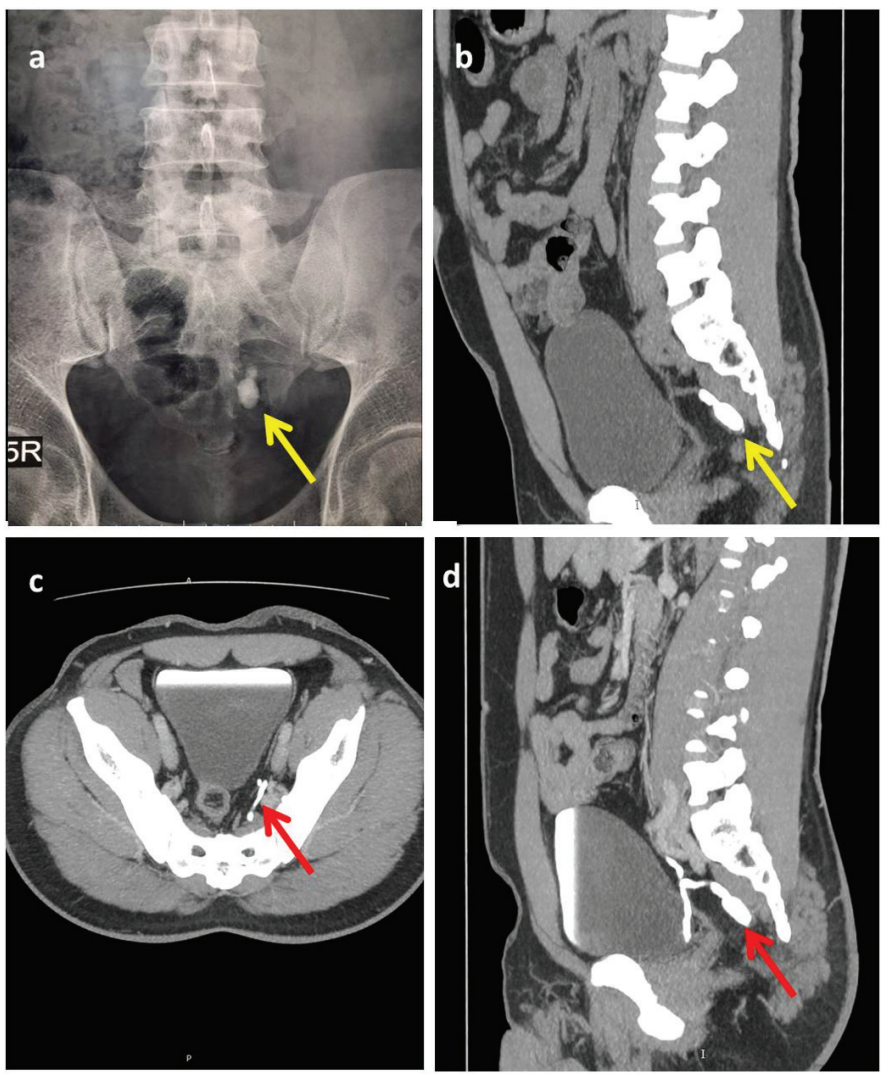

Figure 1. (a) X-ray kidney, ureter, and bladder (KUB), (b) non-contrast computed tomography (CT) KUB showing stones (yellow arrow), (c) CT urogram sagittal view, and (d) axial view showing the diverticulum (red arrow)

acquired form can be subdivided into traction or pulsion types. The pulsing type is usually caused by a distal obstructing stone, and there is an associated proximal hydroureter (5). It may occur after the ureteric wall is weakened by stones, infections, shock wave lithotripsy, or ureteric surgery $(5,6)$. Based on the histopathological findings by Kretschmer (7), the congenital type was described as a blind-ending duplication of ureteric buds. On histopathological evaluation, the diverticulum of this patient most likely represented an abortive ureteral duplication because of its long tubular nature, and all layers of the ureteric wall were preserved. Pulsion or traction types of diverticula are much shorter with a wide neck.

Few previous reports have described the formation of calculi inside the diverticulum $(4,5)$. This may have been due to stasis of the refluxing urine inside the diverticulum or recurrent infections. In the present case, the postero-inferior direction of the distal part of the diverticulum may have led to dependency and urine stasis (Figures 2c and 2d). The stone formation can lead to both congenital and acquired diverticula. CT urogram can identify the exact anatomical relations of the diverticulum to avoid inadvertent damage to nearby vital structures and associated anomalies of the urinary tract. This is more relevant in diverticula with stones, as the anatomical direction of the


Figure 2. (a) Resected specimen with calculi (white arrow) and (b) microscopic view of the diverticular wall (hematoxylin and eosin staining, $\times 100$ )

diverticulum could be different from the expected normal course of a duplex ureter, leading to dependent segments at risk of stasis. The reasons for the unusual anatomy of congenital ureteric diverticula when compared with duplex ureters are unclear. The origin of the diverticulum at a lower level of the ureter, its length, and stone formation in the blind end of the diverticulum were unique features of the present case.

Diverticula are treated depending on the symptoms and associated complications. A conservative approach can be considered in asymptomatic cases and cases diagnosed incidentally on imaging (1). Depending on the configuration of the diverticulum and associated ureteric obstruction, diverticulectomy with or without reconstruction of the ureter has been used as treatment $(2,6)$. This patient underwent ligation and resection of the diverticulum at its junction with the ureter, without the need for reconstruction as the neck was narrow. Resection of the diverticula can be performed laparoscopically if the anatomy of the diverticulum makes it feasible (8).

In conclusion, radiologically visible opacities suggestive of stones located away from the normal course of the ureter can be calculi located in a ureteric diverticulum. These calculi require diverticulectomy with or without ureteric reconstruction. Although congenital ureteric diverticula are formed following an abortive ureteric bud, its anatomy could be completely different 
from that of a duplex ureter. This should be appreciated during surgery to avoid potential complications.

\section{Ethics}

Informed Consent: Informed written consent for publication of the text and accompanying images was obtained from the patient.

Peer-review: Externally peer-reviewed.

\section{Authorship Contributions}

Concept: O.B., A.A., Design: O.B., Data Collection or Processing: O.B., H.S., M.W., M.A., S.T., A.A., Literature Search: O.B., H.S., M.W., M.A., A.A., Writing: O.B., S.T.

Conflict of Interest: No conflict of interest was declared by the authors.

Financial Disclosure: The authors declared that this study received no financial support.

\section{References}

1. Bǔlov VM, Sencha AN. [Ureteral diverticulum]. Urologiia 2010:63-65.

2. Pratt JG, Gahagan HG, Fischman Jl. Diverticulum of the ureter: a review of the literature and a report of two additional cases. J Urol 1947;58:322-326.

3. Roodhooft AM, Boven K, Gentens P, Van Acker KJ. Abdominal colic due to ureteric diverticulum with stone formation. Pediatr Radiol 1987;17:252253.

4. Harrison GS. Transitional cell carcinoma in a congenital ureteral diverticulum. J Urol 1983;129:1231-1232.

5. Yoshida S, Nakagomi K, Goto S. [A case of true ureteral diverticulum]. Nihon Hinyokika Gakkai Zasshi 2005;96:25-28.

6. Aragona F, Bassi P, Passerini Glazel G, Pagano F. Acquired intravesical ureteral diverticulum: an unusual late complication of ureteroneocystostomy. J Urol 1989;141:1420-1421.

7. Kretschmer HL. Duplication of the ureters at their distal ends, one pair ending blindly: so-called diverticula of the ureters. J Urol 1933;30:61-73.

8. Negoro H, Inoue T, Imai K, Goto T, Sawada A, Akamatsu S, Saito R, Kobayashi T, Yamasaki T, Ogawa O. Laparoscopic excision of an acquired ureteral diverticulum: A case report. Asian J Endosc Surg 2019;12:478-481. 